\title{
Human helminthes intestinal parasites of Morang district, Nepal
}

\author{
Shiv Narayan Yadav \\ Department of Zoology, M.M.A.M. Campus, T.U., Biratnagar, Nepal
}

\begin{abstract}
The present studyy examined association between $\mathrm{ABO}$ human blood group and helminth parasites. In total 3000 people were investigated and 1300 individuals were found infected with helminth parasites, namely Ascaris lumbricoides (52.46\%) Ancylostoma duodenale (45.00\%), Trichuris trichura $(1.90 \%)$, Enterobius vermicularis $(0.03 \%)$, Taenia solium $(0.015 \%)$, Hymenolepis nana $(0.15 \%)$.
\end{abstract}

Key words: $\mathrm{ABO}$ blood group, helminthiasis, prevalence rate, random sampling, stool samples

\section{Introduction}

Gastro-intestinal helminthiasis is an important cause of morbidity worldwide and affects a large proportion of human beings. Parasite induced cell reaction causes inflammation as a result of destruction of the host cell. The cell damage causes the secretion of histamine that dilates capillaries to increase blood flow to the damaged sites. An increase in number of eosinophils is one of the symptoms of the helminth infection.

A report of the First World War indicated that ABO system of different populations of the allied armies varied from one population to another. The important point to understand is that the most racial groups includes all types of the $\mathrm{ABO}$ system $\mathrm{A}, \mathrm{B}, \mathrm{O}$ or $\mathrm{AB}$ but in different proportions. These blood groups are permanent trait of an individual. Climate, illness or medical treatment cannot alter the groups which are inherited from parent. One example of frequency (\%) of blood groups $\mathrm{O}, \mathrm{A}, \mathrm{B}$ and $\mathrm{AB}$ of different populations reveals English 45.8, 42.2, 8.7 and 3.2 Russian 32.9, 35.6, 23.2 and 8.1 Chinese 45.5, 22.6, 25.0 and 6.1 and Hindus 30.2, 24.5, 37.2, 8.1, respectively.

Reported the percentage frequencies of blood groups among the blood donor 909 individuals in which Welsh family names and non-Welsh family names showed for 'O' 52.7 and 46.6 for 'A' 35.0 and 42.0 for 'B' 9.7 and 8.3 for 'AB' 2.6 and 3.2. Gupta (1968) found in Bihar 21.55\% individuals in A group 38.34\% individuals in B group, $8.6 \%$ individuals in AB and $31.45 \%$ individuals in $\mathrm{O}$ group. He found no significant sex difference among male and female sexes. It has been examined the blood group of 339 cases of schistosomal hepatic fibrosis in Egypt. There was a higher incidence of blood group 'A' in the schistosoma cases (22\%) with corresponding lower incidence of group 'O' (21.1\%) (Clemens et al., 1989).

\section{Materials and Methods}

By random sampling methods 3000 individuals were studied both for ABO blood group and the occurrence of intestinal helminth parasites in rural areas of different VDCs of Morang district, eastern, Nepal during Jan 2013 to December 2013. 


\section{Routine stool examination techniques}

Stool samples were collected randomly in the screw capped containers with applicators sticks were taken and helminthes parasites were observed by direct smear method.

\section{Routine ABO blood group examination techniques}

The four types of $\mathrm{A}, \mathrm{B}, \mathrm{AB}$ and $\mathrm{O}$ blood group have different agglutinating properties to determine the blood group types of difference persons.. On a glass slide a drop of type A- serum (containing anti-B antibodies) is placed along with a separate drop of type-B serum (containing anti-A antibodies). Where a drop of $\mathrm{O}$ blood group is added to each drop there is no agglutination of $\mathrm{RBCs}$ in either cases. This shows that $\mathrm{O}$ blood group has neither $\mathrm{A}$ nor $\mathrm{B}$ antigen. In general, type-A RBCs are agglutinated by type-B serum and AB type RBCs are agglutinated by both sera.

\section{Results and Discussion}

Occurrence of intestinal helminthes parasites in different $\mathrm{ABO}$ blood groups have been shown in table 1. Out of 3000 individuals of total ABO blood grouping samples, 1700 (56.66\%) were uninfected and 1300 (43.33\%) were infected with helminth parasites.

Table 1. Occurrence of intestinal parasites in different ABO-blood group.

\begin{tabular}{cccccccc}
\hline Blood & Total no & \multicolumn{3}{c}{ Uninfected sample } & \multicolumn{3}{c}{ Infected sample } \\
\cline { 3 - 8 } group & examined & Male & Female & Total & Male & Female & Total \\
\hline $\mathrm{O}$ & 880 & 300 & 240 & 540 & 175 & 165 & 340 \\
& & $(34.09 \%)$ & $(27.27 \%)$ & $(61.36 \%)$ & $(19.88 \%)$ & $(18.75 \%)$ & $(38.63 \%)$ \\
\hline $\mathrm{A}$ & 865 & 290 & 230 & 520 & 200 & 145 & 345 \\
& & $(33.52 \%)$ & $(26.59 \%)$ & $(60.11 \%)$ & $23.12 \%$ & $16.76 \%$ & $39.88 \%$ \\
\hline $\mathrm{B}$ & 933 & 275 & 220 & 495 & 225 & 213 & 438 \\
& & $(29.48 \%)$ & $(23.57 \%)$ & $(53.05 \%)$ & $(24.11 \%)$ & $(22.82 \%)$ & $(46.93 \%)$ \\
\hline $\mathrm{AB}$ & 322 & 100 & 45 & 145 & 110 & 67 & 177 \\
& & $(31.05 \%)$ & $(13.98 \%)$ & $(45.03 \%)$ & $(34.16 \%)$ & $(20.80 \%)$ & $(54.96 \%)$ \\
\hline Total & 3000 & - & - & 1700 & - & - & 1300 \\
& & & & $(56.66 \%)$ & & & $(43.33 \%)$ \\
\hline
\end{tabular}

Among the population under 'O' blood groups, a total 880 out of 340 infected were male 175 (19.08\%) and female 165(18.75\%). Among the specific helminthes parasitic infections on 'O' blood groups are Ascaris lumbricoides (55.43\%) and Ancylostoma duodenale (44.6\%) in male while in female are Ascaris lumbricoides (55.75\%), hookworm (42.84\%) and Trichuris trichura (1.87\%). A blood group, a total 865 out of 345 infested had male 200(23.12\%) and female 145 (16.75\%); and among male are A. lumbricoides 111 (55.5\%), Ancylostoma duodenale 88 $(44.0 \%)$ and $H$. nana $1(0.5 \%)$ while in female roundworm $45(31.03 \%)$, Hookworm 90 $(62.06 \%)$ and T. trichura $10(6.82 \%)$.

B blood group- Among 933, out of 438 are infected and its male 225(24.11\%) are R. W. 125(55.55\%), hookworm 98(43.55\%), Enterobius vermicularis 2(0.88\%) while it's female 213 $(22.82 \%)$ are roundworm $108(51.12 \%)$, hookworm 91(42.72\%), T.t. 12(5.6\%), E.v. $2(0.98 \%)$ 
AB- blood group- Among 322, out of 177 are infested and its male 110(34.16\%) are R. W. $62(56.36 \%)$, A.d. 46(41.81\%), T. solium $2(1.81 \%)$ while in female $67(20.8 \%)$ are R. W. (62.67\%), A.d. 24 (35.82\%) and H. nana 1 (1.4\%).

The prevalence rate of total infection was higher in males $(54.61 \%)$ as compared to female $(45.39 \%)$. Thus Chi $\left(\mathrm{X}^{2}\right)$ square test was employed to determine the association between individuals of $\mathrm{ABO}$ blood system and the occurrence of intestinal helminth parasites. The experimental Chi-square $\left(\mathrm{X}^{2}\right)$ at 0.05 level of significant with 3 degree of freedom was less than the computed value $X^{2}=34.37$. Therefore it was concluded that there is significant difference between infected and uninfected samples and the impact of the ABO blood group on the occurrence of these intestinal helminth parasites. Therefore, the null hypothesis is rejected.

Area of habitation was the major factor determining helminthes prevalence infections rate of most helminthes was higher in males than females. These finding were statically significant. Intestinal helminth parasites is transmitted in males to be higher by the access to health care and education, by the absence of clean water sanitation and irregular use of foot wear. In the rural areas, the 4 blood groups shared the same environment and were of similar nutritional status and were likely to be exposed to similar levels of infections with intestinal helminthes as well as other infectious diseases.

The present result is different from that of the investigators (Gupta, 1968). Due to the environment and nutritional status, area of habitation and socio-economic status were also poor. This study illustrates the importance of controlling for socioeconomic circumstances in epidemiological investigations of helminthiasis. Area of habitation was a good indicator of both socio-economic factors and the level of geohelminths transmission and prevalence.

\section{References}

Barnes, G.L. \& R. Kay. 1977. Blood group and giardiasis. Lancet 1: 808.

Clemens. J.D., D.A. Sack, J.R. Harris, J. Chackraborthi, M.R. Khan, Hudas, F. Ahmed, J. Gomes, M.R. Rao, A.M. Svennerholm, \& J. Holmgren. 1989. ABO blood group and cholera: new observation on specificity of risk and modification of vaccine efficiency. Journal of infectious diseases 159:770-773.

Gupta, R. 1968. Blood group distribution. Pat. J. Med. 42: 1. 